\title{
Induction of seed germination in Orobanche spp. by extracts of traditional Chinese medicinal herbs
}

\author{
MA YongQing ${ }^{1,2 *}$, ZHANG Wei ${ }^{1}$, DONG ShuQi ${ }^{1}$, REN XiangXiang ${ }^{3}$, AN Yu ${ }^{1} \&$ LANG Ming ${ }^{1}$ \\ ${ }^{1}$ College of Resources and Environment, Northwest A \& F University, Yangling 712100, China; \\ ${ }^{2}$ State Key Laboratory of Soil Erosion and Dryland Farming on the Loess Plateau, Institute of Soil and Water Conservation, Northwest \\ A \& F University, Yangling 712100, China; \\ ${ }^{3}$ College of Forestry, Northwest A \& F University, Yangling 712100, China
}

Received September 23, 2011; accepted March 5, 2012

\begin{abstract}
The co-evolution of Orobanche spp. and their hosts within the same environment has resulted in a high degree of adaptation and effective parasitism whereby the host releases parasite germination stimulants, which are likely to be unstable in the soil. Our objective was to investigate whether extracts from non-host plants, specifically, Chinese medicinal plants, could stimulate germination of Orobanche spp. Samples of 606 Chinese medicinal herb species were extracted with deionized water and methanol. The extracts were used to induce germination of three Orobanche species; Orobanche minor, Orobanche cumana, and Orobanche aegyptiaca. O. minor exhibited a wide range of germination responses to the various herbal extracts. $O$. cuma$n a$ and $O$. aegyptiaca exhibited an intermediate germination response to the herbal extracts. $O$. minor, which has a narrow host spectrum, showed higher germination rates in response to different herbal extracts compared with those of $O$. cumana and $O$. aegyptiaca, which have a broader host spectrum. Methanolic extracts of many Chinese herbal species effectively stimulated seed germination among the Orobanche spp., even though they were not the typical hosts. The effective herbs represent interesting examples of potential trap crops. Different countries can also screen extracts from indigenous herbaceous plants for their ability to induce germination of Orobanche spp. seeds. The use of such species as trap plants could diminish the global soil seed bank of Orobanche.
\end{abstract}

Orobanche spp., methanolic extracts, deionized water extracts, germination, Chinese medicinal plants

Citation: Ma Y Q, Zhang W, Dong S Q, et al. Induction of seed germination in Orobanche spp. by extracts of traditional Chinese medicinal herbs. Sci China Life Sci, 2012, 55: 250-260, doi: 10.1007/s11427-012-4302-2

Orobanche spp. are holoparasites of higher plants. Parasitic weeds of the Orobanchaceae family are widely distributed throughout the Mediterranean region, western Asia, and eastern Europe, and they cause substantial losses to global crop production. They are considered to be the most important parasitic weeds on a world-wide scale [1]. There are 11 species of the genus Orobanche in northern and southwestern China [2-4]. The genus is particularly prevalent in the Xinjiang Uygur Autonomous Region, where it has caused yield losses to several economically important plants

*Corresponding author (email: mayongqing@ms.iswc.ac.cn) such as sunflower (Helianthus annuus L.), tobacco (Nicotiana tabacum L.), and tomato (Lycopersicon esculentum Mill.).

Orobanche spp. are difficult weeds to control because of their complex life cycle. There are several discrete steps in the life cycle; the production of a large number of seeds that require a post-ripening period as well as warm and moist conditions, induction of germination by host-derived stimulants, haustorial initiation by host-plant haustorium inducers, attachment to the host root and penetration, establishment of contact with host vascular system, subterranean development, emergence, and flowering. The most serious damage 
to host crops occurs underground before emergence, as described by Parker and Riches [1]. Thus, the depletion of its soil seed bank by inducing "suicidal germination" would be an efficient way to control Orobanche.

Investigations into another parasitic weed, Striga, have advanced research on Orobanche seed germination stimulants. The first compound isolated as a Striga germination stimulant was strigol, later classified as one of a group of plant hormones known as strigolactones. Strigol was isolated from a non-host cotton (Gossypium hirsutum Linn.) plant in the 1960s and 1970s [5,6]. Strigolactones have been extensively characterized as seed germination stimulants of parasitic weeds. They are exuded from plant roots and released into the rhizosphere [7]. To date, three classes of plant secondary metabolites have been shown to induce seed germination in Striga and Orobanche; dihydrosorgoleones, sesquiterpene lactones, and strigolactones [8-10]. Among these, strigolactones show the strongest activity. Different strigolactones have been isolated from host and non-host plants of Orobanche, Phelipanche, and Striga $[10,11]$. To date, the structures of nine strigolactones have been elucidated. These include sorgomol [12], isolated from root exudates of sorghum (Sorghum bicolor L.), and fabacyl acetate [13], isolated from root exudates of pea (Pisum sativum L.). Didehydroorobanchol, orobanchol, orobanchyl acetate, and 5-deoxystrigol were also identified from the root exudates of Fabaceae plants [14]. Research on the isolation and identification of orobanchol $[15,16]$, the first Orobanche germination stimulant isolated from root exudates of red clover (Trifolium pratense L.), demonstrated that strigolactones stimulate germination in both Striga and Orobanche spp. Recently, Evidente et al. [17] reported the isolation of two strigolactone-like compounds (peagol and peagoldione) from pea root exudates. Both compounds showed selective stimulation of Orobanche seed germination.

Much attention has been paid to the isolation and identification of germination stimulants from host plants of Striga and Orobanche. Although the first germination stimulant, strigol, was isolated from a non-host cotton plant, most attempts to isolate germination stimulants have been made using host plants. Fernández-Apariciol et al. [18] reported the induction of seed germination of nine Orobanche and Pheliphanche species by root exudates of 41 plants. However, most of these plants were cultivated field crops rather than wild plants.

We hypothesized that, if host plants could exude stable germination stimulants, many of the parasitic seeds would be induced to germinate during the growing season and this would greatly reduce the soil seed bank. In theory, this would reduce or eliminate the parasite problem, but it is unknown whether this strategy is successful in practice. Therefore, it is important to identify potent germination stimulants from non-host plants. This raises the question of where to find such plant materials, since not all plants are able to produce the stimulants. It is an established practice in Chinese medicine to use medicinal herbs to cure human and animal diseases. We speculated that because these plants contain substances with anti-fungal and/or antibacterial activity, and because they did not co-evolve with Orobanche plants, they may produce alternative chemicals to the known strigolactones that might be stable in soil.

Previously, we reported the screening of extracts from 383 traditional Chinese medicinal herbs and tested their ability to induce or inhibit the germination of Striga hermonthica seeds [19]. Deionized water and methanolic extracts of more than 26 herbs induced germination of $S$. hermonthica [19]. It was also reported that the Chinese medicinal herbs Menispermum dauricum (DC.) and Houttuynia cordata Thunb. produced germination stimulants for $S$. hermonthica [20-22]. Jin et al. [23] reported the extraction of active substances from 240 Chinese medicinal herbs and found that more than 40 of them were able to induce seed germination of $O$. minor. Therefore, we assumed that there would be other Chinese herbs that could produce germination stimulants for Orobanche spp. weeds. To test this hypothesis, we evaluated 606 economically important and commonly used Chinese medicinal herbal extracts for their efficacy to stimulate seed germination in Orobanche minor, Orobanche cumana, and Orobanche aegyptiaca.

\section{Materials and methods}

\subsection{Seeds and chemicals}

Seeds of $O$. cumana and $O$. aegyptiaca were collected in 2008/2009 from infested sunflower (H. annuus) and tomato (L. esculentum) fields in Xinjiang Uygur Autonomous Region, China. Seeds of $O$. minor were provided by Professor Koichi Yoneyama (Utsunomiya University, Japan). The germination stimulant, GR24, a synthetic analogue of strigol, was provided by Professor Binne Zwanenburg (University of Nijmegen, The Netherlands).

\subsection{Herbal powders}

We obtained 606 species of Chinese medicinal herbs from Chinese medicinal drug stores distributed in all the provinces of mainland China, excluding Tibet. The medicinally useful parts of the herbs were air-dried and then milled using a herbal medicine disintegrator (FW135-177, Tianjin Taisite Instrument Co., Ltd., Tianjin, China) and passed through a sieve $(0.45 \mathrm{~mm}$ in diameter $)$.

\subsection{Surface sterilization and conditioning of Orobanche seeds}

All seeds were surface-sterilized by immersion for $3 \mathrm{~min}$ in $1 \%$ sodium hypochlorite. Seeds of $O$. minor were then thoroughly washed with deionized water, pre-treated (con- 
ditioned) for 6-8 d on $8 \mathrm{~mm}$ disks of glass fiber filter paper (GFFP, Whatman, GE Healthcare UK Ltd., Buckinghamshire, UK), and then incubated in the dark at $25^{\circ} \mathrm{C}$. Seeds of $O$. cumana and $O$. aegyptiaca were already receptive before conditioning based on preliminary testing and the results of Plakhine et al. [24]; hence these seeds were used directly for bioassays.

\subsection{Germination assay}

A sample $(100 \mathrm{mg})$ of each herb was sonicated for $30 \mathrm{~min}$ in $1.5 \mathrm{~mL}$ deionized water. The aqueous extracts were centrifuged in a microcentrifuge (Millipore Cat. No. XX42 CF0, 60 Lot No. N8JMB042A, Nihon Millipore Ltd. Yonezawa, Japan). We prepared three concentrations of the supernatants; undiluted, and 10- and 100-fold dilutions in deionized water. The two aqueous solutions and the undiluted extracts were assayed immediately by applying $15 \mu \mathrm{L}$ of each solution to the conditioned Orobanche seeds on $8 \mathrm{~mm}$ disks of GFFP in Petri dishes. The disks with the conditioned seeds were briefly blotted on filter paper (Shuangquan GB/T19142007, Hangzhou Wohua Filter Paper Co., Ltd., Hangzhou, China) to remove excess water before treatments with the extracts. Three concentrations of methanolic extracts were obtained in the same way. Aliquots $(15 \mu \mathrm{L})$ of the test solution were applied to 8 -mm disks of GFFP and the disks were allowed to dry. A disk with conditioned Orobanche seeds was placed on top of the dried treated disk and moistened with $30 \mu \mathrm{L}$ deionized water. The treated seeds were incubated in the dark at $25^{\circ} \mathrm{C}$ and examined for germination 8 days later. Controls comprised three GR24 treated disks with conditioned Orobanche seeds (positive control) and three deionized water treated disks (negative control).

Individual treatments were replicated four times. We used 20 herbs in each batch of bioassay experiments. The herbal extracts shown to affect Orobanche seed germination were included in the next round of germination testing.
When the screening of the 606 herbs was complete, the ones that positively affected Orobanche seed germination were retested using the same group of conditioned seeds. Germinated and non-germinated seeds were counted under a binocular dissecting microscope at $20 \times$ magnification. Seeds were considered to have germinated on protrusion of the "germ-tube" from the seed coat.

\subsection{Statistical analysis}

Orobanche spp. germination data were subjected to analysis of variance. Tukey's multiple range tests were used to separate the means. Data were processed using Excel 2007 and DPS 6.55 software (DPS Soft Inc., Hangzhou, China).

\section{Results}

Seeds of all the three Orobanche spp. germinated at a high rate $(>78 \%)$ when treated with GR24. None of them germinated when treated with deionized water. In this study, only those herb species whose extracts induced more than $30 \%$ of Orobanche seeds to germinate at one or more of the concentrations (undiluted, 10- , or 100-fold dilutions) were considered.

\subsection{Induction of germination of Orobanche minor seeds}

Deionized water extracts of 20 herbal species, at at least one concentration, resulted in high germination rates $(>60 \%)$ of O. minor. The maximum germination rate of $90.3 \%$ was induced by the 10-fold dilution of the extract of Juncus effusus L. var. decipiens Buchen. Most of the undiluted extracts induced germination at lower rates. However, the undiluted J. effusus L. var. decipiens Buchen extract induced germination at the rate $72.7 \%$ (Table 1 ).

Based on our results, we classified the herbs into three

Table 1 Germination of $O$. minor seeds induced by deionized water extracts of traditional Chinese medicinal herbs $(\%)^{\text {a) }}$

\begin{tabular}{|c|c|c|c|}
\hline Medicinal herbs & Undiluted & 10-fold dilution & 100-fold dilution \\
\hline Aconitum carmichaeli Debx. & $0.0 \mathrm{~g}^{\dagger}$ & 24.7 cdefg & 41.5 abcdefgh \\
\hline Akebia quinata (Thunb.) Decne. & $0.0 \mathrm{~g}$ & 59.4 abcdef & $65.8 \mathrm{abc}$ \\
\hline Aloe vera $\mathrm{L}$. & $21.9 \mathrm{~cd}$ & 51.7 abcdefg & 50.2 abcdefg \\
\hline Alpinia katsumadai Hayata. & $0.0 \mathrm{~g}$ & 63.7 abcde & $62.9 \mathrm{abcd}$ \\
\hline Angelica sinensis (Oliv.) Diels & $0.0 \mathrm{~g}$ & 25.2 cdefg & 40.0 abcdefgh \\
\hline Ardisia japonica (Hornsted) Blume. & $0.0 \mathrm{~g}$ & 14.7 efg & 47.6 abcdefgh \\
\hline Arenaria kansuensis Maxim. & $0.0 \mathrm{~g}$ & 52.7 abcdefg & 45.7 abcdefgh \\
\hline Artemisia argyi Levl. et Vant. & $7.7 \mathrm{efg}$ & 43.9 abcdefg & $64.2 \mathrm{abcd}$ \\
\hline Artemisiae anomalae Herba. & $0.0 \mathrm{~g}$ & 46.0 abcdefg & 27.7 abcdefgh \\
\hline Benincasa hispida (Thunb.) Cogn. & $0.0 \mathrm{~g}$ & 36.8 bcdefg & 20.9 cdefgh \\
\hline Blumea balsamifera DC. & $0.0 \mathrm{~g}$ & $10.2 \mathrm{fg}$ & 45.5 abcdefgh \\
\hline Buddleja officinalis Maxim. & $0.0 \mathrm{~g}$ & 31.5 bcdefg & 30.1 abcdefgh \\
\hline Cannablis sativa $\mathrm{L}$. & $8.0 \mathrm{efg}$ & 66.3 abcde & $5.6 \mathrm{gh}$ \\
\hline
\end{tabular}




\begin{tabular}{|c|c|c|c|}
\hline Medicinal herbs & Undiluted & 10-fold dilution & 100-fold dilution \\
\hline Chrysanthemum indicum $\mathrm{L}$. & $0.0 \mathrm{~g}$ & 25.8 cdefg & 39.2 abcdefgh \\
\hline Cibotium barometz (L.) J. Sm. & $0.0 \mathrm{~g}$ & 48.4 abcdefg & 54.2 abcdef \\
\hline Cinnamomum cassia Presl & $0.0 \mathrm{~g}$ & 40.4 abcdefg & 32.9 abcdefgh \\
\hline Citrus aurantium L. & $0.0 \mathrm{~g}$ & $0.0 \mathrm{~g}$ & 43.3 abcdefgh \\
\hline Citrus reticulata Blanco & $0.0 \mathrm{~g}$ & $15.4 \mathrm{efg}$ & 49.4 abcdefg \\
\hline Citrus tangerina Hort. et Tanaka & $0.0 \mathrm{~g}$ & $0.0 \mathrm{~g}$ & 34.6 abcdefgh \\
\hline Cyathula officinalis Kuan. & $0.0 \mathrm{~g}$ & 40.1 abcdefg & $72.2 \mathrm{ab}$ \\
\hline Cynanchum paniculatum (Bunge) Kitagawa & $0.0 \mathrm{~g}$ & 59.4 abcdef & $65.8 \mathrm{abc}$ \\
\hline Dalbergia odorifera T. Chen. & $21.7 \mathrm{~cd}$ & $79.3 \mathrm{ab}$ & $61.7 \mathrm{abcd}$ \\
\hline Epimedium brevicornum Maxim. & $0.0 \mathrm{~g}$ & 33.0 bcdefg & 39.1 abcdefgh \\
\hline Eriocaulon buergerianum Koern. & $14.0 \mathrm{def}$ & 41.7 abcdefg & 24.3 bcdefgh \\
\hline Eupatorium fortunei Turcz. & $0.0 \mathrm{~g}$ & 48.4 abcdefg & $61.3 \mathrm{abcd}$ \\
\hline Farfugium japonicum (L.) Kitam & 15.3 de & $37.1 \mathrm{bcdefg}$ & 9.9 efgh \\
\hline Glycine $\max$ (L.) Merr. & $0.0 \mathrm{~g}$ & 33.6 bcdefg & 33.6 abcdefgh \\
\hline Helianthus annuus L. & $0.0 \mathrm{~g}$ & 55.2 abcdef & 51.7 abcdefg \\
\hline Ilex rotunda Thunb. & $2.5 \mathrm{fg}$ & 16.5 defg & $66.9 \mathrm{abc}$ \\
\hline Illicium verum Hook. f. & $0.0 \mathrm{~g}$ & $8.6 \mathrm{fg}$ & $70.3 \mathrm{ab}$ \\
\hline Jasminum sambac (L.) Ait. & $44.7 \mathrm{~b}$ & 27.8 bcdefg & 34.2 abcdefgh \\
\hline Juncus effusus L. var. decipiens Buchen & $72.7 \mathrm{a}$ & $90.3 \mathrm{a}$ & 29.0 abcdefgh \\
\hline Kaempferia galanga $\mathrm{L}$. & $0.0 \mathrm{~g}$ & 44.8 abcdefg & 36.8 abcdefgh \\
\hline Ligusticum chuanxiong Hort & $0.0 \mathrm{~g}$ & 38.1 abcdefg & $62.9 \mathrm{abcd}$ \\
\hline Lophatherum gracile Bongn. & $0.0 \mathrm{~g}$ & 67.3 abcde & 28.7 abcdefgh \\
\hline Lycopodium clavatum $\mathrm{L}$. & $5.6 \mathrm{efg}$ & 44.9 abcdefg & 9.0 efgh \\
\hline Magnolia officinalis Rehd. et Wils. & $0.0 \mathrm{~g}$ & $9.8 \mathrm{fg}$ & 33.7 abcdefgh \\
\hline Nardostachys chinensis Batal. & $16.0 \mathrm{de}$ & 37.2 bcdefg & 35.7 abcdefgh \\
\hline Nelumbo nucifera Gaertn. & $2.0 \mathrm{fg}$ & $69.0 \mathrm{abcd}$ & $72.0 \mathrm{ab}$ \\
\hline Ottelia alismoides (L.) Pers. & $0.0 \mathrm{~g}$ & 30.4 bcdefg & 25.4 bcdefgh \\
\hline Paeonia lactiflora Pall. & $0.0 \mathrm{~g}$ & 50.6 abcdefg & 54.8 abcdef \\
\hline Phyllanthus urinaria Linn. & $0.0 \mathrm{~g}$ & $15.3 \mathrm{efg}$ & 43.5 abcdefgh \\
\hline Phyllostachys sulphurea (Carr.) A. et C. Riv. & $0.0 \mathrm{~g}$ & 41.8 abcdefg & $74.5 \mathrm{a}$ \\
\hline Pinellia pedatisecta Schott. & $48.4 \mathrm{~b}$ & 59.1 abcdef & 24.7 bcdefgh \\
\hline Poeonia suffruticosa Andr. & $0.0 \mathrm{~g}$ & 29.6 bcdefg & 55.3 abcde \\
\hline Polygonum orientale L. & $0.0 \mathrm{~g}$ & 17.5 defg & 33.9 abcdefgh \\
\hline Polyporus umbellatus (Pers.) Fries & $47.0 \mathrm{~b}$ & 38.2 abcdefg & 45.2 abcdefgh \\
\hline Poria $\operatorname{cocos}$ (Schw.) Wolf. & $29.3 \mathrm{c}$ & 40.4 abcdefg & 16.5 defgh \\
\hline Prunus mume Sieb. et. Zucc. & $0.0 \mathrm{~g}$ & 44.6 abcdefgl & 8.9 efgh \\
\hline Pteris multifida Poir. & $0.0 \mathrm{~g}$ & 35.5 bcdefg & $0.0 \mathrm{~h}$ \\
\hline Pueraria lobata (Willd.) Ohwi . & $0.0 \mathrm{~g}$ & 44.2 abcdefg & 20.7 cdefgh \\
\hline Pulsatilla chinensis (Bge.) Reg. & $0.0 \mathrm{~g}$ & $0.0 \mathrm{~g}$ & 50.7 abcdefg \\
\hline Pyrola calliantha $\mathrm{H}$. Andres. & $0.0 \mathrm{~g}$ & 20.3 cdefg & 31.9 abcdefgh \\
\hline Pyrrosia lingua (Thunb.) Farw. & $0.0 \mathrm{~g}$ & 48.5 abcdefg & 27.5 abcdefgh \\
\hline Rhaponticum uniflorum (L.) DC & 4.0 efg & 54.0 abcdef & $7.3 \mathrm{fgh}$ \\
\hline Rheum forrestii Diels. & $0.0 \mathrm{~g}$ & 33.0 bcdefg & $62.2 \mathrm{abcd}$ \\
\hline Rhododendron molle (B1.) G. Don & $0.0 \mathrm{~g}$ & 54.3 abcdef & 44.7 abcdefgh \\
\hline Rhus chinensis Mill. & $0.0 \mathrm{~g}$ & 39.0 abcdefg & $0.0 \mathrm{~h}$ \\
\hline Sargentodoxa cuneata (oliv.) Rehd. et Wils. & $0.0 \mathrm{~g}$ & 37.1 bcdefg & 20.1 cdefgh \\
\hline Saruma henryi Oliv. & $0.0 \mathrm{~g}$ & 42.1 abcdefg & 38.0 abcdefgh \\
\hline Saussurea costus (Falc.) Lipsch. & $0.0 \mathrm{~g}$ & 28.2 bcdefg & $62.2 \mathrm{abcd}$ \\
\hline Scrophularia ningpoensis Hemsl. & $0.0 \mathrm{~g}$ & 41.5 abcdefg & 53.3 abcdefg \\
\hline Scutellaria barbata D. Don & $0.0 \mathrm{~g}$ & 25.6 cdefg & 32.6 abcdefgh \\
\hline Senecio scandens Buch.-Ham. & $0.0 \mathrm{~g}$ & 29.1 bcdefg & 50.4 abcdefg \\
\hline Setaria viridis (L.) Beauv. & $0.0 \mathrm{~g}$ & 50.7 abcdefg & $61.9 \mathrm{abcd}$ \\
\hline Stemona japonica (BL.) Miq. & 5.0 efg & 44.5 abcdefg & 47.4 abcdefgh \\
\hline Sterculia scaphigera Wall. & $0.0 \mathrm{~g}$ & 38.7 abcdefg & $0 \mathrm{~h}$ \\
\hline Tinospora sinensis (Lour.) Merr. & $0.0 \mathrm{~g}$ & $72.4 \mathrm{abc}$ & 31.6 abcdefgh \\
\hline Vaccaria segetalis (Neck.) Garcke & 8.3 efg & 65.0 abcde & 40.5 abcdefgh \\
\hline Viola yedoensis Mak. & $0.0 \mathrm{~g}$ & $0.0 \mathrm{~g}$ & 54.6 abcdef \\
\hline
\end{tabular}

a) $\dagger$, Means in the same column followed by different letters differ significantly according to Tukey's multiple range tests $(P<0.05)$. $O$. minor germination rate in deionized water was $0.0 \%$. 
groups according to their ability to induce germination of Orobanche seeds. The first group consisted of those species whose undiluted extracts induced little or no germination, their 10-fold dilution induced a high germination rate $(>60 \%)$, and their 100-fold dilution induced a lower germination rate. This group included Cannablis sativa L.; Dalbergia odorifera T. Chen., Lophatherum gracile Bongn., Tinospora sinensis (Lour.) Merr., and Vaccaria segetalis (Neck.) Garcke (Table 1).

The second group comprised herbs whose undiluted water extracts induced little or no germination of $O$. minor seeds, but their dilutions induced higher germination rates with greater dilution. This group included Aconitum carmichaeli Debx., Akebia quinata (Thunb.) Decne., Angelica sinensis (Oliv.) Diels, Ardisia japonica (Hornsted) Blume., Artemisia argyi Levl. et Vant., Blumea balsamifera DC., Chrysanthemum indicum L., Cibotium barometz (L.) J. Sm., Citrus aurantium L., Citrus reticulata Blanco, Citrus tangerina Hort. et Tanaka, Cyathula officinalis Kuan., Cynanchum paniculatum (Bunge) Kitagawa, Epimedium brevicornum Maxim., Eupatorium fortunei Turcz., Ilex rotunda Thunb., Illicium verum Hook. f., Ligusticum chuanxiong Hort, Magnolia officinalis Rehd. et Wils., Phyllanthus urinaria Linn., Phyllostachys sulphurea (Carr.) A. et C. Riv., Poeonia suffruticosa Andr., Polygonum orientale L., Pulsatilla chinensis (Bge.) Reg., Pyrola calliantha H. Andres., Rheum forrestii Diels., Saussurea costus (Falc.) Lipsch., Scrophularia ningpoensis Hemsl., Scutellaria barbata D. Don, Senecio scandens Buch.-Ham., Setaria viridis (L.) Beauv., and Viola yedoensis Mak. (Table 1). This group of herbs has the potential for further purification of germination stimulants or could be considered as trap plants. The results indicated that these extracts contained high concentrations of highly active substances.

The third group included herbs whose undiluted extracts resulted in little or no germination of $O$. minor seeds, and whose 10- and 100-fold-diluted extracts induced similar levels of germination. The species in this group included Aloe vera L., Alpinia katsumadai Hayata., Arenaria kansuensis Maxim., Buddleja officinalis Maxim., Glycine max (L.) Merr., Helianthus annuus L., Nardostachys chinensis Batal., Nelumbo nucifera Gaertn., Paeonia lactiflora Pall., Saruma henryi Oliv. and Stemona japonica (BL.) Miq. (Table 1).

The methanolic extracts of 100 herbs induced seed germination in $O$. minor (Table 2). Compared with deionized water extracts, there were more undiluted methanolic extracts that induced germination of $O$. minor seeds. Germination rates of more than $60 \%$ were observed for undiluted methanolic extracts from Fritillaria cirrhosa D. Don, Ginkgo biloba L., Oryza sativa L., Pinellia ternate (Thunb.) Breit and V. segetalis (Neck.) Garcke. However, when the extracts from these five herbs were diluted, all showed a sharp reduction in their ability to induce germination. Induction of germination by methanolic extracts of other herbs was similar to that of deionized water extracts, and could also be similarly divided into three groups. There were 47 herbs for which both the deionized water and methanolic extracts induced seed germination. Of these, five herbs induced a germination rate of more than $60 \%$ when supplied as dilutions of either deionized water or methanolic extracts. These five herbs were A. katsumadai Hayata., A. argyi Levl. et Vant., E. fortunei Turcz., L. gracile Bongn. and V. segetalis (Neck.) Garcke (Tables 1 and 2). Further research should be conducted on those herbs that showed germination induction ability only as a methanolic extract, with greater activity as the dilution increased. These included Abrus fruticulosus Wall. ex wight et Arn, Albizzia julibrissin Durazz, Elsholtzia splendens Nakai ex F. Maekawa, Selaginella tamariscina (Beauv.) Spring, and Soroseris umbrella (Franch.) Stebb. Further dilutions of their methanolic extracts should be tested for their ability to induce germination. This may reveal different compounds from those in the deionized water extracts.

\subsection{Induction of germination of Orobanche cumana seeds}

There were lower rates of germination of $O$. cumana seeds, compared with those of $O$. minor. The water extracts of only 18 species stimulated germination rates exceeding $30 \%$ (Table 3). Extracts from Peucedanum praeruptorum Dunn. and $S$. viridis (L.) Beauv. induced germination rates of $49.2 \%$ and $48.8 \%$, respectively. The water extracts of six herbs, Areca catechu L., A. kansuensis Maxim., C. indicum L., P. urinaria Linn., Polyporus umbellatus (Pers.) Fries. and $S$. viridis (Linn.) Beauv., stimulated germination of both $O$. cumana and $O$. minor seeds. Similarly, methanolic extracts of only 16 herb species stimulated $O$. cumana seed germination at rates exceeding $30 \%$ (Table 4) although methanolic extracts of Sterculia scaphigera Wall. induced germination rates greater than $60 \%$. Methanolic extracts of C. paniculatum (Bunge) Kitagawa, H. annuus L., J. effusus L. var. decipiens Buchen, Prunus mume Sieb. et Zucc., and $S$. scaphigera Wall. stimulated germination of both $O$. minor and $O$. cumana seeds (Tables 2 and 4), and their deionized water extracts also induced germination of $O$. minor seeds (Tables 1 and 4).

\subsection{Induction of germination of Orobanche aegyptiaca seeds}

Seed germination rates of $O$. aegyptiaca in response to herbal extracts were also lower than those of $O$. minor. Deionized water extracts of only 21 out of 606 herbs induced O. aegyptiaca seeds to germinate. The maximum germination rate $(81.9 \%)$ was induced by the 10 -fold dilution of Hedyotis diffusa Willd. (Table 5). Deionized water extracts of four herbs, A. kansuensis Maxim., C. indicum L., P. urinaria Linn., and $S$. viridis (L.) Beauv., induced germination of all three Orobanche spp. (Tables 1, 3, and 5). Deionized 
Table 2 Germination of $O$. minor seeds induced by methanolic extracts of traditional Chinese medicinal herbs $(\%)^{\text {a) }}$

\begin{tabular}{|c|c|c|c|}
\hline Medicinal herbs & Undiluted & 10-fold dilution & 100-fold dilution \\
\hline Abrus fruticulosus Wall. ex wight et Arn & $0.0 \mathrm{p}$ & 37.3 abcdefgh & 54.0 abcde \\
\hline Aconitum carmichaeli Debx. & 35.0 bcdefghijklmn & 22.0 defgh & $5.6 \mathrm{lmn}$ \\
\hline Aconitum coreanum (Lévl.) Raipaics & 41.9 bcdefghijk & 38.7 abcdefgh & 28.7 abcdefghijklmn \\
\hline Acorus gramineus Soland. & 2.9 nop & 36.3 abcdefgh & $9.8 \mathrm{ijklmn}$ \\
\hline Ailanthus altissima (Mill.) Swingle. & $0.0 \mathrm{p}$ & 60.3 abcdef & $0.0 \mathrm{n}$ \\
\hline Akebia quinata (Thunb.) Decne. & $0.0 \mathrm{p}$ & $17.0 \mathrm{fgh}$ & 34.8 abcdefghijklmn \\
\hline Aloe vera $\mathrm{L}$. & $0.0 \mathrm{p}$ & 27.4 cdefgh & 35.8 abcdefghijklmn \\
\hline Alpinia katsumadai Hayata. & 30.7 cdefghijklmnop & 64.7 abcde & 50.1 abcdefghi \\
\hline Amomum tsao-ko Crevost et lem. & $53.0 \mathrm{abcd}$ & 29.3 abcdefgh & 10.0 ijklmn \\
\hline Arctium lappa $\mathrm{L}$. & $12.5 \mathrm{jklmnop}$ & 45.8 abcdefgh & $6.1 \mathrm{lmn}$ \\
\hline Ardisia japonica (Hornsted) Blume. & $0.0 \mathrm{p}$ & 40.3 abcdefgh & 39.2 abcdefghijklmn \\
\hline Arenaria kansuensis Maxim. & 36.2 bcdefghijklm & 41.7 abcdefgh & 48.6 abcdefghij \\
\hline Artemisia apiacea Hance. & $0.0 \mathrm{p}$ & 34.3 abcdefgh & 34.9 abcdefghijklmn \\
\hline Artemisia argyi Lévl. et Vant & 25.3 defghijklmnop & 62.0 abcdef & 33.7 abcdefghijklmn \\
\hline Artemisia capillaris Thunb. & $0.0 \mathrm{p}$ & 55.7 abcdefg & 18.5 abcdefghijklmn \\
\hline Benincasa hispida (Thunb.) Cogn. & $0.0 \mathrm{p}$ & 36.5 abcdefgh & 52.9 abcdef \\
\hline Blumea balsamifera DC. & $0.0 \mathrm{p}$ & 24.8 defgh & 42.9 abcdefghijklm \\
\hline Cannabis sativa $\mathrm{L}$. & 16.4 ghijklmnop & 41.8 abcdefgh & 32.5 abcdefghijklmn \\
\hline Cassia obtusifolia L. & 18.6 efghijklmnop & 51.7 abcdefgh & 46.5 abcdefghijkl \\
\hline Cibotium barometz (L.) J. Sm. & $0.0 \mathrm{p}$ & 43.0 abcdefgh & 11.6 ghijklmn \\
\hline Cimicifuga foetida $\mathrm{L}$. & $0.0 \mathrm{p}$ & 37.2 abcdefgh & $4.9 \mathrm{mn}$ \\
\hline Citrus aurantium $\mathrm{L}$. & $0.0 \mathrm{p}$ & 35.1 abcdefgh & 41.6 abcdefghijklm \\
\hline Citrus sinensis (L.) Osbeck & $0.0 \mathrm{p}$ & 42.6 abcdefgh & 35.5 abcdefghijklmn \\
\hline Citrus tangerina Hort. et Tanaka & 8.9 lmnop & 42.3 abcdefgh & 15.4 defghijklmn \\
\hline Cremastra variabilis (BI.) Nakai & 45.2 abcdefghi & 25.7 cdefgh & $4.8 \mathrm{mn}$ \\
\hline Curcuma longa $\mathrm{L}$. & 21.0 defghijklmnop & $66.7 \mathrm{abcd}$ & $8.0 \mathrm{jklmn}$ \\
\hline Cyperus rotundus $\mathrm{L}$. & $0.0 \mathrm{p}$ & 46.9 abcdefgh & $3.9 \mathrm{mn}$ \\
\hline Dalbergia odorifera T. Chen. & $0.0 \mathrm{p}$ & 27.1 cdefgh & 38.0 abcdefghijklmn \\
\hline Dichroa febrifuga Lour. & 28.5 defghijklmnop & 26.9 cdefgh & 50.0 abcdefghi \\
\hline Dioscorea hypoglauca Palib & 13.7 hijklmnop & $72.7 \mathrm{abc}$ & 13.0 efghijklmn \\
\hline Elsholtzia splendens Nakai ex F. Maekawa & $0.0 \mathrm{p}$ & 36.2 abcdefgh & 52.9 abdef \\
\hline Eupatorium fortunei Turcz. & $0.0 \mathrm{p}$ & 42.9 abcdefgh & $66.3 \mathrm{a}$ \\
\hline Euphoria longan (Lour.) Steud. & $0.0 \mathrm{p}$ & 46.5 abcdefgh & $2.7 \mathrm{mn}$ \\
\hline Farfugium japonicum (L.) Kitam & 39.2 bcdefghijkl & 20.5 defgh & $4.1 \mathrm{mn}$ \\
\hline Fritillaria cirrhosa D. Don & $61.3 \mathrm{abc}$ & 58.0 abcdefg & 10.7 hijklmn \\
\hline Gardenia jasminoides Ellis. & $0.0 \mathrm{p}$ & 47.3 abcdefgh & 28.4 abcdefghijklmn \\
\hline Ginkgo biloba $\mathrm{L}$. & $63.9 \mathrm{ab}$ & 22.9 defgh & $7.3 \mathrm{klmn}$ \\
\hline Glycine $\max (\mathrm{L}$.$) Merr.$ & $1.3 \mathrm{op}$ & 22.3 defgh & $59.3 \mathrm{ab}$ \\
\hline Helianthus annuus $\mathrm{L}$. & 46.0 abcdefgh & 44.5 abcdefgh & 38.5 abcdefghijklmn \\
\hline Herba Artemisiae Anomalae. & 43.1 abcdefghij & 60.4 abcdef & 19.9 bcdefghijklmn \\
\hline Homalomena occulta (Lour.) Schott & 36.5 bcdefghijklm & 41.1 abcdefgh & 27.8 abcdefghijklmn \\
\hline Ilex rotunda Thunb. & $0.0 \mathrm{p}$ & 44.3 abcdefgh & 31.0 abcdefghijklmn \\
\hline Illicium verum Hook. f. & $0.0 \mathrm{p}$ & 48.4 abcdefgh & $58.8 \mathrm{ab}$ \\
\hline Indigofera tinctoria $\mathrm{L}$. & 47.2 abcdefg & $12.9 \mathrm{gh}$ & $0.0 \mathrm{n}$ \\
\hline Jasminum sambac (L.) Ait. & 34.2 bcdefghijklmn & 38.9 abcdefgh & 20.3 bcdefghijklmn \\
\hline Juncus effusus L. var. decipiens Buchen & 47.7 abcdefg & 53.0 abcdefgh & 52.7 abcdefg \\
\hline Kaempferia galanga $\mathrm{L}$. & $0.0 \mathrm{p}$ & 51.1 abcdefgh & 39.9 abcdefghijklmn \\
\hline Lemna minor $\mathrm{L}$. & 23.8 defghijklmnop & 41.5 abcdefgh & 17.4 cdefghijklmn \\
\hline Lepidium apetalum Willd. & $0.0 \mathrm{p}$ & 44.0 abcdefgh & 42.0 abcdefghijklmn \\
\hline Ligusticum chuanxiong Hort & $0.0 \mathrm{p}$ & 46.5 abcdefgh & $55.1 \mathrm{abcd}$ \\
\hline Lonicera japonica Thunb. & $0.0 \mathrm{p}$ & 41.0 abcdefgh & 14.7 defghijklmn \\
\hline
\end{tabular}




\begin{tabular}{|c|c|c|c|}
\hline Medicinal herbs & Undiluted & 10-fold dilution & 100-fold dilution \\
\hline Lophatherum gracile Bongn. & $0.0 \mathrm{p}$ & $75.2 \mathrm{ab}$ & $66.0 \mathrm{a}$ \\
\hline Lycopodium clavatum $\mathrm{L}$. & 48.8 abcdef & 39.8 abcdefgh & 12.0 fghijklmn \\
\hline Magnolia officinalis Rehd. et Wils. & $0.0 \mathrm{p}$ & 29.7 abcdefgh & 31.2 abcdefghijklmn \\
\hline Morus alba $\mathrm{L}$. & 13.4 ijklmnop & 44.4 abcdefgh & 26.2 abcdefghijklmn \\
\hline Nardostachys chinensis Batal. & $0.0 \mathrm{p}$ & 32.7 abcdefgh & $64.3 \mathrm{a}$ \\
\hline Nelumbo nucifera Gaertn. & 6.4 mnop & 39.2 abcdefgh & $4.8 \mathrm{mn}$ \\
\hline Oryza sativa $\mathrm{L}$ & $74.3 \mathrm{a}$ & 49.7 abcdefgh & 27.0 abcdefghijklmn \\
\hline $\begin{array}{l}\text { Panax pseudo-ginseng Wall. var. notoginseng (Burkill) Hoo } \\
\& \text { Tseng }\end{array}$ & 28.7 defghijklmnop & 50.3 abcdefgh & 51.7 abcdefgh \\
\hline Phyllostachys sulphurea (Carr.) A. et C. Riv. & $0.0 \mathrm{p}$ & 43.8 abcdefgh & 30.6 abcdefghijklmn \\
\hline Physalis alkekengi L. var. franchetii (Mast.) Makino & $0.0 \mathrm{p}$ & 34.1 abcdefgh & 29.3 abcdefghijklmn \\
\hline Pinellia ternata (Thunb.) Breit & $62.0 \mathrm{abc}$ & $7.0 \mathrm{~h}$ & $4.7 \mathrm{mn}$ \\
\hline Polygonum cuspidatum Sieb. et Zucc. & 11.9 jklmnop & 33.8 abcdefgh & $3.4 \mathrm{mn}$ \\
\hline Portulaca oleracea $\mathrm{L}$. & 27.7 defghijklmnop & 61.0 abcdef & 52.7 abcdefg \\
\hline Prunus mume Sieb. et Zucc. & $0.0 \mathrm{p}$ & 26.3 cdefgh & 48.2 abcdefghijk \\
\hline Pueraria lobata (Willd.) Ohwi. & 39.4 bcdefghijkl & 25.4 cdefgh & $6.1 \mathrm{lmn}$ \\
\hline Pulsatilla chinensis (Bge.) Reg. & $0.0 \mathrm{p}$ & 17.7 efgh & 31.8 abcdefghijklmn \\
\hline Punica granatum $\mathrm{L}$. & 10.0 klmnop & 29.3 abcdefgh & $4.0 \mathrm{mn}$ \\
\hline Pyrola calliantha $\mathrm{H}$. Andres. & 6.4 mnop & 32.5 abcdefgh & 25.5 abcdefghijklmn \\
\hline Pyrrosia lingua (Thunb.) Farw. & 24.0 defghijklmnop & 49.9 abcdefgh & 28.5 abcdefghijklmn \\
\hline Ranunculus japonicus Thunb. & $0.0 \mathrm{p}$ & 32.4 abcdefgh & 22.9 bcdefghijklmn \\
\hline Ranunculus ternatus Thunb. & 48.8 abcdef & 51.4 abcdefgh & 10.7 hijklmn \\
\hline Rhaponticum uniflorum (L.) DC. & 46.5 abcdefg & 44.2 abcdefgh & 14.9 defghijklmn \\
\hline Rheum forrestii Diels. & 42.0 bcdefghijk & $6.8 \mathrm{~h}$ & $6.5 \mathrm{lmn}$ \\
\hline Rhododendron molle (Bl.) G. Don & $0.0 \mathrm{p}$ & 40.2 abcdefgh & $8.9 \mathrm{jklm}$ \\
\hline Rubia cordifolia $\mathrm{L}$. & 4.9 mnop & 44.2 abcdefgh & $7.4 \mathrm{klmn}$ \\
\hline Sargassum siliguastrum (Turn.) C. Ag. & 50.1 abcde & 24.6 defgh & $0.0 \mathrm{n}$ \\
\hline Scrophularia ningpoensis Hemsl. & $0.0 \mathrm{p}$ & 53.9 abcdefgh & $54.3 \mathrm{abcd}$ \\
\hline Scutellaria barbata D. Don. & 21.7 defghijklmnop & 40.0 abcdefgh & 29.5 abcdefghijklmn \\
\hline Selaginella tamariscina (Beauv.) Spring. & $0.0 \mathrm{p}$ & 28.4 bcdefgh & 34.4 abcdefghijklmn \\
\hline Semiaquilegia adoxoides (DC.) Mak. & 21.0 defghijklmnop & 57.7 abcdefgh & 22.7 bcdefghijklmn \\
\hline Sesamum indicum DC. & $0.0 \mathrm{p}$ & 63.7 abcdef & $9.3 \mathrm{ijklmn}$ \\
\hline Setaria viridis (L.) Beauv. & $0.0 \mathrm{p}$ & 41.5 abcdefgh & 34.0 abcdefghijklmn \\
\hline Soroseris umbrella (Franch.) Stebb. & 17.0 fghijklmnop & 34.4 abcdefgh & 48.3 abcdefghijk \\
\hline Speranskia tuberculata (Bge.) Baill. & 15.7 ghijklmnop & 44.7 abcdefgh & $8.2 \mathrm{jklmn}$ \\
\hline Stemona japonica (BL.) Miq. & $0.0 \mathrm{p}$ & $76.3 \mathrm{a}$ & $4.3 \mathrm{mn}$ \\
\hline Stephania tetrandra S. Moore. & 14.1 hijklmnop & 42.5 abcdefgh & $8.2 \mathrm{jklmn}$ \\
\hline Sterculia scaphigera Wall. & 19.8 efghijklmnop & 50.5 abcdefgh & $56.8 \mathrm{abc}$ \\
\hline Tribulus terrestris $\mathrm{L}$. & $0.0 \mathrm{p}$ & 36.5 abcdefgh & 39.5 abcdefghijklmn \\
\hline Trichosanthes kirilowii Maxim. & 35.0 bcdefghijklmn & 22.9 defgh & 20.0 bcdefghijklmn \\
\hline Vaccaria segetalis (Neck.) Garcke & $64.0 \mathrm{ab}$ & 54.3 abcdefgh & $7.7 \mathrm{jklmn}$ \\
\hline Vatica mangachapoi Blauco. & $0.0 \mathrm{p}$ & 46.2 abcdefgh & 37.7 abcdefghijklmn \\
\hline Viola yedoensis Mak. & $0.0 \mathrm{p}$ & 35.2 abcdefgh & 41.6 abcdefghijklm \\
\hline
\end{tabular}

a) $O$. minor germination rate in deionized water was $0.0 \%$.

water extracts of Benincasa hispida (Thunb.) Cogn., C. paniculatum (Bunge) Kitagawa, $H$. annuus L. Pueraria lobata (Willd.) Ohwi and S. viridis (L.) Beauv. induced germination of both $O$. aegyptiaca and O. minor seeds (Tables 1 and 5). Deionized water extracts of Artemisia capillaris Thunb., A. kansuensis Maxim., C. indicum L., Elsholtzia ciliata (Thunb.) Hyland., P. praeruptorum Dunn., P. urinaria Linn., S. viridis (L.) Beauv. and Tamarix chinensis Lour. induced germination of both $O$. aegyptiaca and $O$. cumana seeds (Tables 3 and 5).

Among the methanolic extracts, the maximum germination rate $(69.6 \%)$ was induced by the extract from $S$. scaphigera Wall. Similar to water extracts, methanolic extracts of only 20 species induced germination of $O$. aegyptiaca seeds. Only the methanol extract of Lonicera japonica Thunb. at 100 -fold dilution induced a germination rate greater than 
Table 3 Germination of $O$. cumana seeds induced by deionized water extracts of traditional Chinese medicinal herbs $(\%)^{\text {a) }}$

\begin{tabular}{|c|c|c|c|}
\hline Medicinal herb & Undiluted & 10-fold dilution & 100-fold dilution \\
\hline Achyranthes aspera Linn. & $0.0 \mathrm{~d}$ & $35.3 \mathrm{a}$ & $0.0 \mathrm{c}$ \\
\hline Alpinia officinarum Hance & $0.0 \mathrm{~d}$ & $39.3 \mathrm{a}$ & $0.0 \mathrm{c}$ \\
\hline Amomum cardamomum $\mathrm{L}$. & $0.0 \mathrm{~d}$ & $34.2 \mathrm{a}$ & $0.0 \mathrm{c}$ \\
\hline Antenoron filiforme (Thunb.) Rob. et Vaut. & $28.3 \mathrm{bc}$ & $39.4 \mathrm{a}$ & $0.0 \mathrm{c}$ \\
\hline Areca catechu $\mathrm{L}$. & $0.0 \mathrm{~d}$ & $33.6 \mathrm{a}$ & $23.3 \mathrm{ab}$ \\
\hline Arenaria kansuensis Maxim. & $20.1 \mathrm{c}$ & $36.7 \mathrm{a}$ & $0.0 \mathrm{c}$ \\
\hline Artemisia capillaris Thunb. & $40.3 \mathrm{ab}$ & $21.9 \mathrm{ab}$ & $0.0 \mathrm{c}$ \\
\hline Atractylodes macrocephala Koidz. & $0.0 \mathrm{~d}$ & $0.0 \mathrm{~b}$ & $30.9 \mathrm{ab}$ \\
\hline Chrysanthemum indicum $\mathrm{L}$. & $0.0 \mathrm{~d}$ & $33.2 \mathrm{a}$ & $41.2 \mathrm{a}$ \\
\hline Elsholtzia ciliata (Thunb.) Hyland. & $0.0 \mathrm{~d}$ & $47.4 \mathrm{a}$ & $0.0 \mathrm{c}$ \\
\hline Gleditsia sinensis Lam. & $53.6 \mathrm{a}$ & $39.1 \mathrm{a}$ & $22.4 \mathrm{~b}$ \\
\hline Pelargonium hortorum Bailey & $0.0 \mathrm{~d}$ & $35.4 \mathrm{a}$ & $20.1 \mathrm{~b}$ \\
\hline Peucedanum praeruptorum Dunn. & $0.0 \mathrm{~d}$ & $49.2 \mathrm{a}$ & $0.0 \mathrm{c}$ \\
\hline Pharbitis nil (L.) Choisy. & $0.0 \mathrm{~d}$ & $30.0 \mathrm{ab}$ & $0.0 \mathrm{c}$ \\
\hline Phyllanthus urinaria Linn. & $0.0 \mathrm{~d}$ & $40.6 \mathrm{a}$ & $24.5 \mathrm{ab}$ \\
\hline Polyporus umbellatus (Pers.) Fries. & $25.8 \mathrm{c}$ & $32.5 \mathrm{a}$ & $0.0 \mathrm{c}$ \\
\hline Setaria viridis (L.) Beauv. & $20.8 \mathrm{c}$ & $48.8 \mathrm{a}$ & $0.0 \mathrm{c}$ \\
\hline Tamarix chinensis Lour. & $0.0 \mathrm{~d}$ & $42.5 \mathrm{a}$ & $12.7 \mathrm{bc}$ \\
\hline
\end{tabular}

a) O. cumana germination rate in deionized water was $0.0 \%$.

Table 4 Germination of $O$. cumana seeds induced by methanolic extracts of traditional Chinese medicinal herbs $(\%)^{\text {a) }}$

\begin{tabular}{|c|c|c|c|}
\hline Medicinal herbs & Undiluted & 10-fold dilution & 100-fold dilution \\
\hline Antenoron filiforme (Thunb.) Rob. et Vaut. & $58.9 \mathrm{a}$ & $51.5 \mathrm{ab}$ & $0.0 \mathrm{~d}$ \\
\hline Crotalaria ferruginea Grah. & $52.7 \mathrm{ab}$ & $45.0 \mathrm{ab}$ & $0.0 \mathrm{~d}$ \\
\hline Cynanchum paniculatum (Bunge) Kitagawa & $0.0 \mathrm{~d}$ & $56.9 \mathrm{ab}$ & $10.5 \mathrm{~cd}$ \\
\hline Diospyros kaki L. f. & $50.9 \mathrm{abc}$ & $8.1 \mathrm{c}$ & $0.0 \mathrm{~d}$ \\
\hline Emilia sonchifolia (Linn.) DC. & $7.5 \mathrm{~d}$ & $55.7 \mathrm{ab}$ & $36.1 \mathrm{abcd}$ \\
\hline Glehnia littoralis F. Schmidt ex Miq. & $39.2 \mathrm{abcd}$ & $26.5 \mathrm{bc}$ & $17.8 \mathrm{bcd}$ \\
\hline Juncus effusus L. var. decipiens Buchen & $38.3 \mathrm{abcd}$ & $52.8 \mathrm{ab}$ & $50.3 \mathrm{abc}$ \\
\hline Liquidambar formosana Hance & $42.2 \mathrm{abcd}$ & $58.3 \mathrm{a}$ & 40.4 abcd \\
\hline Murdannia divergens (C. B. Clarke.) Bruckn. & $0.0 \mathrm{~d}$ & $56.7 \mathrm{ab}$ & $0.0 \mathrm{~d}$ \\
\hline Pelargonium hortorum Bailey & $12.9 \mathrm{bcd}$ & $0.0 \mathrm{c}$ & $55.6 \mathrm{ab}$ \\
\hline Picrasma quassioides (D. Don) Benn & $52.0 \mathrm{abc}$ & $43.9 \mathrm{ab}$ & $0.0 \mathrm{~d}$ \\
\hline Prunus mume Sieb. et Zucc. & $0.0 \mathrm{~d}$ & $53.9 \mathrm{ab}$ & $37.6 \mathrm{abcd}$ \\
\hline Scutellaria baicalensis Georgi & $0.0 \mathrm{~d}$ & $58.4 \mathrm{a}$ & $52.8 \mathrm{ab}$ \\
\hline Sterculia scaphigera Wall. & $9.5 \mathrm{~cd}$ & $60.8 \mathrm{a}$ & $63.6 \mathrm{a}$ \\
\hline
\end{tabular}

a) O. cumana germination rate in deionized water was $0.0 \%$.

$50 \%$; others induced germination at rates lower than $50 \%$ (Table 6).

\section{Discussion}

It is widely believed that seeds of Orobanche spp. require a conditioning period of several days under suitable temperature and moisture conditions before germinating in response to germination stimulants $[1,25-27]$. We found that $O$. minor seeds indeed required a conditioning period before responding to germination stimulants (herbal extracts). This finding was consistent with other reports [24]. However, seeds of O. cumana and O. aegyptiaca, which infested sunflower and tomato fields in the Xinjiang Uygur Autonomous Region of China, did not require a preconditioning treatment. They germinated directly when treated with GR24 or medicinal herbal extracts, which is consistent with the findings of more recent studies [24,28].

Some undiluted herbal extracts did not induce germination. This may have been because the concentration of the stimulant was too high, or because germination inhibitors were present. In some cases, a 10-fold dilution was sufficient to overcome the inhibiting effects and allow the germination stimulant to induce seed germination. When the germination rate decreased with the 100 -fold dilution, it is 
Table 5 Germination of $O$. aegyptiaca seeds induced by deionized water extracts of traditional Chinese medicinal herbs $(\%)^{\text {a) }}$

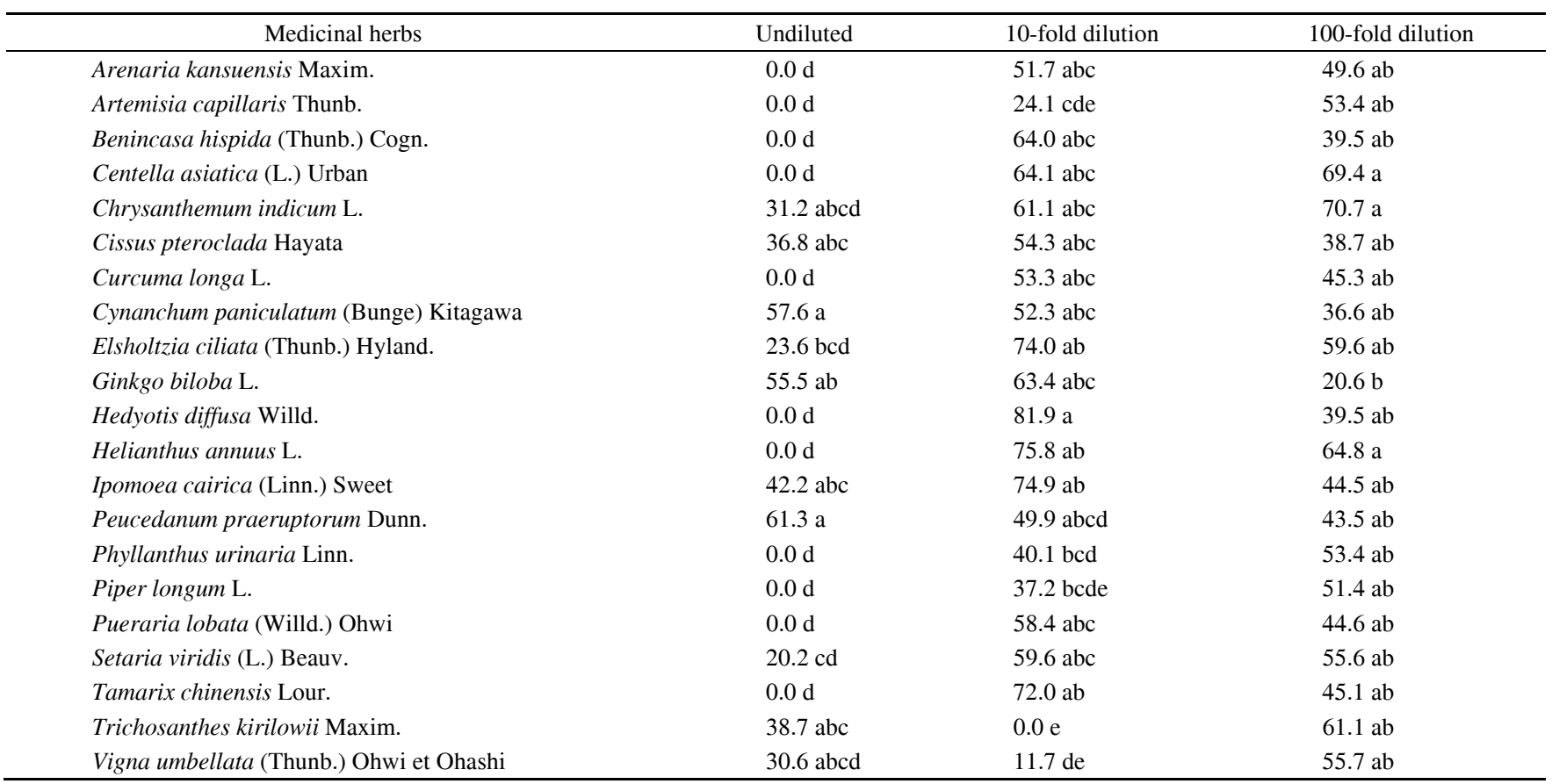

a) O. aegyptiaca germination rate in deionized water was $0.0 \%$.

Table 6 Germination of $O$. aegyptiaca seeds induced by methanolic extracts of traditional Chinese medicinal herbs $(\%)^{\text {a) }}$

\begin{tabular}{|c|c|c|c|}
\hline Medicinal herbs & Undiluted & 10-fold dilution & 100-fold dilution \\
\hline Antenoron filiforme (Thunb.) Rob. et Vaut. & $66.4 \mathrm{ab}$ & $49.2 \mathrm{a}$ & $19.7 \mathrm{abc}$ \\
\hline Blumea balsamifera DC. & $0.0 \mathrm{e}$ & $29.4 \mathrm{a}$ & $41.4 \mathrm{ab}$ \\
\hline Buddleja officinalis Maxim. & 29.8 bcde & $40.4 \mathrm{a}$ & $28.2 \mathrm{abc}$ \\
\hline Citrus aurantium $\mathrm{L}$. & 19.3 cde & $40.8 \mathrm{a}$ & $37.6 \mathrm{ab}$ \\
\hline Crotalaria ferruginea Grah. & $55.6 \mathrm{abc}$ & $41.1 \mathrm{a}$ & $44.0 \mathrm{ab}$ \\
\hline Cynanchum paniculatum (Bunge) Kitagawa & $39.7 \mathrm{abcd}$ & $44.7 \mathrm{a}$ & $18.4 \mathrm{bc}$ \\
\hline Dianthus superbus Linn. & $22.0 \mathrm{cde}$ & $32.1 \mathrm{a}$ & $24.3 \mathrm{abc}$ \\
\hline Fructus tritici Levis & 26.2 cde & $31.2 \mathrm{a}$ & $15.0 \mathrm{bc}$ \\
\hline Ginkgo biloba $\mathrm{L}$. & $16.1 \mathrm{de}$ & $30.0 \mathrm{a}$ & $22.7 \mathrm{abc}$ \\
\hline Glycine $\max (\mathrm{L}$.$) Merr.$ & $0.0 \mathrm{e}$ & $43.8 \mathrm{a}$ & $43.1 \mathrm{ab}$ \\
\hline Helianthus annuus L. & 36.3 abcde & $38.4 \mathrm{a}$ & $22.2 \mathrm{abc}$ \\
\hline Ipomoea cairica (Linn.) Sweet & 36.6 abcde & $27.3 \mathrm{a}$ & $23.2 \mathrm{abc}$ \\
\hline Lobelia chinensis Lour. & $49.0 \mathrm{abcd}$ & $33.5 \mathrm{a}$ & $4.4 \mathrm{c}$ \\
\hline Lonicera japonica Thunb. & 20.4 cde & $34.0 \mathrm{a}$ & $51.9 \mathrm{a}$ \\
\hline Momordica grosvenori Swingle & 24.0 cde & $31.2 \mathrm{a}$ & $27.5 \mathrm{abc}$ \\
\hline Murdannia divergens (C. B. Clarke.) Bruckn. & $0.0 \mathrm{e}$ & $40.1 \mathrm{a}$ & $22.5 \mathrm{abc}$ \\
\hline Picrasma quassioides (D.Don) Benn & $39.2 \mathrm{abcd}$ & $22.7 \mathrm{a}$ & $13.6 \mathrm{bc}$ \\
\hline Setaria italica (L.) Beauv & $14.4 \mathrm{de}$ & $38.0 \mathrm{a}$ & $41.2 \mathrm{ab}$ \\
\hline Sterculia scaphigera Wall. & $69.6 \mathrm{a}$ & $39.7 \mathrm{a}$ & $0.0 \mathrm{c}$ \\
\hline Taxillus chinensis (DC.) Danser & 25.5 cde & $36.5 \mathrm{a}$ & $18.5 \mathrm{bc}$ \\
\hline
\end{tabular}

a) O. aegyptiaca germination rate in deionized water was $0.0 \%$.

likely that the concentration of the stimulant was not high enough in these solutions. Further isolations of germination stimulants should exclude this group of herbs.

The present study on Orobanche germination stimulants has built upon the study of the parasite Striga. One reason for this is because germination stimulants that affected
Striga also affected Orobanche. Isolation, identification, and structural elucidation of compounds that stimulate Stri$g a$ germination from genuine host plants has been given utmost priority for almost 50 years [1]. The primary objective has been to synthesize structural mimics with high activities to induce suicidal germination of the parasite seeds, 
thereby reducing or eliminating the seed bank in the soil. However, isolation of the active compounds has been difficult, as host plants produce very small amounts of these germination stimulants. Most of the germination stimulants isolated and identified so far possess the same basic molecular structure, and are collectively known as strigolactones [7-9,29-32]. To date, more than 14 strigolactones have been identified, mainly from plant root exudates [33].

In our experiment, approximately $20 \%$ of the medicinal herb extracts induced $O$. minor seeds to germinate. This percentage was much lower for germination of $O$. cumana $(<3 \%)$ and 0 . aegyptiaca $(<4 \%)$. These results were consistent with the findings of Fernández-Aparicio et al. [19], and suggested that not all plants produce sufficient amounts of strigolactones to induce germination of Orobanche spp., as also suggested by Akiyama and Hayashi [34] and Klee [35]. In addition, Chinese medicinal herb extracts may contain substances that inhibit the germination-promoting activities of strigolactones. In our previous study on S. hermonthica, water extracts of 27 of the 383 herbs tested (7\%), inhibited germination of $S$. hermonthica seeds, even when GR24 was added before applying the extracts [20]. Only a very small number of medicinal herb extracts induced germination of $O$. cumana and $O$. aegyptiaca seeds (Tables 3-6), disproving the hypothesis that genes involved in strigolactone biosynthesis would be present in all higher plants. It is estimated that no more than $7 \%$ of herbaceous plants (3\% for $O$. cumana and $4 \%$ for $O$. aegyptiaca stimulation) could induce seed germination of $O$. cumana and $O$. aegyptiaca. This leaves unanswered the question, why were most of the herbs unable to induce germination of Orobanche spp.?

To date, there is no definitive proof that the germination of parasitic weed seeds in the field is induced by one single signal compound or class of compounds [7]. In the present experiments, we used synthetic strigol as a positive control (data not shown), and found that it induced high germination rates among the three tested species of Orobanche. Large quantities of long-lived seeds allow the parasite to show a high degree of genetic adaptability to changes in host resistance and cultivation practices. As long as the seed bank is not controlled, the need to control the parasite will persist whenever a susceptible host is grown in an infested field [36].

On the basis of circumstantial evidence and experimental results, we concluded that germination stimulants from susceptible hosts and their synthetic mimics are likely to be unstable in soil [37-39]. As observed in the field, host plants are unable to exude sufficient stable germination stimulants during the growing season, and therefore, few parasitic seeds germinate. This increases the seed bank of the parasite in the soil, leading to a persistent parasite problem. To reduce the seed bank of the parasite in the soil, we must find potent germination stimulants from genuine non-host plants. The question is which plant materials are best suited to meet this objective. We suggest that similar research should now be conducted in Orobanche-infested areas by introducing herbaceous plants that have not co-evolved with the parasites. Introduction of species that can induce germination of Orobanche spp. into affected cropping systems may result in 'suicidal germination' of the weed. This practice would have the additional advantage of producing valuable herbal plants that can improve animal and human health at the same time. Furthermore, because these herbal plants did not co-evolve with the parasites, they may produce and store parasitic seed germination stimulants with completely different molecular structures from those that have already been isolated. From these different stimulants, synthetic structural analogs may be developed that have a high capability to induce suicidal germination of the parasites, decreasing or eliminating their seed reserves in soil.

This work was supported by the National Natural Science Foundation of China (Grant No. 30870403) and State Key Laboratory of Soil Erosion and Dryland Farming on the Loess Plateau (Grant No. 10502-Z7-3). We thank Prof. A. Eneji and Prof. D. Warrington (visiting professor, State Key Laboratory of Soil Erosion and DryLand Farming on the Loess Plateau, Institute of Soil and Water Conservation, Chinese Academy of Sciences and Ministry of Water Resources) for critically reading and editing this manuscript.

1 Parker C, Riches C R. Parasitic Weeds of the World: Biology and Control. Wallingford: CAB International, UK, 1993

2 Ren W Y, Li Y, Ma H X, et al. Study on the effect of Orobanche cumana on main economic properties of sunflower and its control. J Hebei Agr Uni, 1992, 15: 63-66

3 Yu H Y, Xue L J, Qiao Y M, et al. Identification of the newly introduced sunflower germplasm resistance to sunflower broomrape in Jilin Province. J Plant Genetic Resour, 2000, 1: 65-65

4 Wang P D, Yang X, Zhang X W, et al. Preliminary report on sunflower broomrape in Shanxi Province. J Shanxi Agr, 2003, 31: 75-77

5 Cook C E, Whichard L P, Turner B, et al. Germination of witchweed (Striga lutea Lour.): isolation and properties of a potent stimulant. Science, 1966, 154: 1189-1189

6 Cook C E, Whichard L P, Wall M, et al. Germination stimulants. II. Structure of strigol, a potent seed germination stimulant for witchweed (Striga lutea). J Am Chem Soc, 1972, 94: 6198-6199

7 Bouwmeester H J, Matusova R, Zhongkui S, et al. Secondary metabolite signaling in host-parasitic plant interactions. Curr Opin Plant Biol, 2003, 6: 358-364

8 Butler L. Chemical communication between the parasitic weed Striga and its crop host: a new dimension of allelochemistry. In: Inderjit K, Dakshini M M, Einhellig F A, eds. Allelopathy: Organisms, Processes and Applications. Washington, DC: ACS Symposium Series, USA, 1995

9 Wigchert S C M, Zwanenburg B. A critical account on the inception of Striga seed germination. J Agr Food Chem, 1999, 47: 1320-1325

10 Xie X N, Kusumoto D, Takeuchi Y, et al. 2'-Epi-orobanchol and solanacol, two unique strigolactones, germination stimulants for root parasitic weeds, produced by tobacco. J Agr Food Chem, 2007, 55: 8067-8072

11 Yoneyama K, Xie X N, Takeuchi Y. Strigolactones: structures and biological activities. Pest Manag Sci, 2009, 65: 467-470

12 Xie X N, Yoneyama K, Kusumoto D, et al. Isolation and identification of alectrol as (+)-orobanchyl acetate, a germination stimulant for root parasitic plants. Phytochemistry, 2008, 69: 427-431

13 Xie X N, Yoneyama K, Harada Y, et al. Fabacyl acetate, a germination stimulant for root parasitic plants from Pisum sativum. Phyto- 
chemistry, 2009, 70: 211-215

14 Yoneyama K, Xie X N, Sekimoto H, et al. Strigolactones, host recognition signals for root parasitic plants and arbuscular mycorrhizal fungi, from Fabaceae plants. New Phytol, 2008, 179: 484-494

15 Yokota T, Sakai H, Okuno K, et al. Alectrol and orobanchol, germination stimulants for Orobanche minor, from its host red clover. Phytochemistry, 1998, 49: 1967-1973

16 Mori K, Matsui J, Yokota T, et al. Structure and synthesis of orobanchol, the germination stimulant for Orobanche minor. Tetrahedron Lett, 1999, 40: 943-946

17 Evidente A, Fernandez-Aparicio M, Cimmino A, et al. Peagol and peagoldione, two new strigolactone-like metabolites isolated from pea root exudates. Tetrahedron Lett, 2009, 50: 6955-6958

18 Fernández-Aparicio M, Flores F, Rubiales D. Recognition of root exudates by seeds of broomrape (Orobanche and Phelipanche) species. Ann Bot, 2009, 103: 423

19 Ma Y Q, Cheng J M, Inanaga S, et al. Induction and inhibition of Striga hermonthica (Del.) Benth. germination by extracts of traditional Chinese medicinal herbs. Agron J, 2004, 96: 1349-1356

20 Ma Y Q, Babiker A G T, Suginoto Y, et al. Effect of medium composition on production of Striga hermonthica (Del.) Benth germination stimulant(s) by Menispermum dauricum (DC.) root cultures. J Agr Food Chem, 1998, 46: 1587-1592

21 Ma Y Q, Babiker A G T, Ali I A, et al. Striga hermonthica (Del.) Benth germination stimulant (s) from Menispermum dauricum (DC.) root culture. J Agr Food Chem, 1996, 44: 3355-3359

22 Ma Y Q, Shui J, Inanage S, et al. Stimulatory effects of Houttuynia cordata Thunb. on seed germination of Striga hermonthica (Del.) Benth. Allelopathy J, 2005, 15: 49-56

23 Jin F P, Hua G H, Ma Y Q, et al. Effect of traditional Chinese medicinal herb extracts on Orobanche minor seed germination. Acta Bot Boreal Occident Sin, 2008, 28: 77-83

24 Plakhine D, Ziadna H, Joel D M. Is seed conditioning essential for Orobanche germination? Pest Manag Sci, 2009, 65: 492-496

25 Pieterse A. The broomrapes (Orobanchaceae): a review. Abstr Trop Agric, 1979, 5: 9-35

26 Hsiao A I, Worsham A D, Moreland D E. Effects of temperature and dl-strigol on seed conditioning and germination of witchweed (Striga asiatica). Ann Bot, 1988, 61: 65-72

27 Kebreab E, Murdoch A J. A quantitative model for loss of primary dormancy and induction of secondary dormancy in imbibed seeds of
Orobanche spp. J Exp Bot, 1999, 50: 211-219

28 Plakhine D, Joel D M. Ecophysiological consideration of Orobanche cumana germination. Helia, 2010, 33: 13-18

29 Yoneyama K, Takeuchi Y, Yokota T. Production of clover broomrape seed germination stimulants by red clover root requires nitrate but is inhibited by phosphate and ammonium. Physiol Plantarum, 2001, 112: 25-30

30 Sato D, Awad A A, Chae S H, et al. Analysis of strigolactones, germination stimulants for Striga and Orobanche, by high-performance liquid chromatography/tandem mass spectrometry. J Agr Food Chem, 2003, 51: 1162-1168

31 Sato D, Awad A A, Takeuchi Y, et al. Confirmation and quantification of strigolactones, germination stimulants for root parasitic plants Striga and Orobanche, produced by cotton. Biosci Biotechnol Biochem, 2005, 69: 98-102

32 Rani K, Zwanenburg B, Sugimoto Y, et al. Biosynthetic considerations could assist the structure elucidation of host plant produced rhizosphere signalling compounds (strigolactones) for arbuscular mycorrhizal fungi and parasitic plants. Plant Physio Bioch, 2008, 46: 617-626

33 Yoneyama K, Awad A A, Xie X N, et al. Strigolactones as germination stimulants for root parasitic plants strigolactones as germination stimulants for root parasitic plants. Plant Cell Physiol, 2010, 51: 1095-1103

34 Akiyama K, Hayashi H. Strigolactones: chemical signals for fungal symbionts and parasitic weeds in plant roots. Ann Bot, 2006, 97: 925-931

35 Klee H. Plant biology: hormones branch out. Nature, 2008, 455: $176-177$

36 Rubiales D, Fernández-Aparicio M, Wegmann K, et al. Revisiting strategies for reducing the seed bank of Orobanche and Phelipanche spp. Weed Res, 2009, 49: 23-33

37 Babiker A, Hamdoun A M, Rudwan A, et al. Influence of soil moisture on activity and persistence of the strigol analogue GR 24. Weed Res, 1987, 27: 173-178

38 Fate G, Chang M, Lynn D G. Control of germination in Striga asiatica: chemistry of spatial definition. Plant Physiol, 1990, 93: 201-207

39 Joel D M, Stelfens J C, Matthews D E. Germination of weedy root parasites. In: Kigel J, Galili G, eds. Seed Development and Germination. New York: Maecel Dekker, Inc., USA, 1995

Open Access This article is distributed under the terms of the Creative Commons Attribution License which permits any use, distribution, and reproduction in any medium, provided the original author(s) and source are credited. 